\title{
On the continuous spectral component of the Floquet operator for a periodically kicked quantum system
}

\author{
James McCaw* and B. H. J. McKellar ${ }^{\dagger}$ \\ School of Physics, Research Centre for High Energy Physics, \\ The University of Melbourne, Victoria, 3010, Australia.
}

(Dated: October 19, 2018)

\begin{abstract}
By a straightforward generalisation, we extend the work of Combescure [J. Stat. Phys. 59, 679 (1990)] from rank-1 to rank- $N$ perturbations. The requirement for the Floquet operator to be pure point is established and compared to that in Combescure. The result matches that in $\mathrm{McCaw}$ and McKellar [J. Math. Phys. 46, 032108 (2005)]. The method here is an alternative to that work. We show that if the condition for the Floquet operator to be pure point is relaxed, then in the case of the $\delta$-kicked Harmonic oscillator, a singularly continuous component of the Floquet operator spectrum exists. We also provide an in-depth discussion of the conjecture presented in the work of Combescure of the case where the unperturbed Hamiltonian is more general. We link the physics conjecture directly to a number-theoretic conjecture of Vinogradov [The Method of Trigonometrical Sums in the Theory of Numbers (Interscience, London, 1954)] and show that a solution of Vinogradov's conjecture solves the physics conjecture. The result is extended to the rank- $N$ case. The relationship between our work and the work of Bourget [J. Math. Anal. Appl. 276, 28 (2002); 301, 65 (2005)] on the physics conjecture is discussed.
\end{abstract}

*Electronic mail: j.mccaw@physics.unimelb.edu.au

${ }^{\dagger}$ Electronic mail: b.mckellar@physics.unimelb.edu.au 


\section{Introduction}

The spectral analysis of the Floquet operator (the unitary time-evolution operator over a single kick period) is of great interest for periodically perturbed Hamiltonian systems. There are general arguments $[4,5,14,19,21]$ which indicate that an understanding or classification of the spectrum of the time-evolution operator can provide information on the dynamics of the system. In particular, the existence of a singularly continuous spectrum of the Floquet operator allows for a slow diffusive energy growth over time, typical of a chaotic system. Thus, this work has significance in the broad field of quantum chaos. For a more detailed discussion of the links among spectral analysis, dynamics and chaos, see the introductory sections of [19] and references therein.

The work in [19] established a non-perturbative stability result on the spectral nature of the Floquet operator for simple systems with a rank- $N$ perturbation periodic in time. The conditions under which the Floquet spectrum remains pure point were established. Here, we will first show the same result, but in a very different manner, before proceeding to determine when a continuous spectrum may arise. This result sheds further light on the array of possible dynamics that periodically perturbed systems may experience.

We consider Hamiltonians of the form

$$
H(t)=H_{0}+\left(\sum_{k=1}^{N} \lambda_{k}\left|\psi_{k}\right\rangle\left\langle\psi_{k}\right|\right) \sum_{n=0}^{\infty} \delta(t-n T),
$$

where $\lambda_{k} \in \mathbb{R}$ and each vector $\left|\psi_{k}\right\rangle$ is a linear combination of the $H_{0}$ basis states, $\left|\phi_{n}\right\rangle$,

$$
\left|\psi_{k}\right\rangle=\sum_{n=0}^{\infty}\left(a_{k}\right)_{n}\left|\phi_{n}\right\rangle .
$$

The states $\left|\psi_{k}\right\rangle$ are orthogonal

$$
\left\langle\psi_{k} \mid \psi_{l}\right\rangle=\delta_{k l}
$$

The Floquet operator ${ }^{1}$ is

$$
V \equiv U(T)=e^{-i H_{0} T / \hbar} e^{-i\left(\sum_{k} \lambda_{k}\left|\psi_{k}\right\rangle\left\langle\psi_{k}\right|\right) / \hbar}
$$

The basic result, as established in [19] is that if every $\left|\psi_{k}\right\rangle$ is in $l_{1}\left(H_{0}\right)$, the spectrum will remain pure point for almost every perturbation strength.

\footnotetext{
${ }^{1}$ Our Floquet operator differs from that in Combescure [15] and Bourget [7,8]. An erronous $T$ was introduced in [15] and it has been carried through in the literature. Note that the theorems proved therein are not invalidated in any way by this error.
} 
If this condition is dropped for any one of the $\left|\psi_{k}\right\rangle$, then we no longer have $V_{\lambda_{1}, \ldots, \lambda_{N}}$ pure point. In fact, on the subspace $\mathcal{H}_{k}$ defined by that space for which $\left|\psi_{k}\right\rangle$ is a cyclic vector for operator $U$, the spectrum is purely continuous. At this point, we note that Milek and Seba [21] have incorrectly concluded from Combescure's work that the existence of a $\psi$ such that $\psi$ is in the continuous subspace of $\mathcal{H}$ implies that the whole of $\mathcal{H}$ is continuous for the operator $V$. This statement would require the assumption that $\psi$ is a cyclic vector for $U$, which is simply impossible for $|\psi\rangle\langle\psi|$ as an arbitrary projection.

For Milek and Seba's work to be properly justified, we show that a sufficient condition is that Vinogradov's number-theoretic conjecture, stated over fifty year ago, ${ }^{2}$ is true. This observation is linked to the conjecture put forward by Combescure [15] and partially addressed by Bourget [7]. After the completion of this work, we became aware of a recent paper by Bourget [8] which successfully resolves the issues with Milek and Seba's work by building on the earlier work in [7]. Bourget's new work in no way invalidates the arguments presented herethe two approaches are complimentary.

In Section II we extend Combescure's rank-1 theorem on the pure point spectral nature of $V$ to the rank- $N$ case. In Section III we then show the existence of a continuous spectrum for the case where $H_{0}$ is the harmonic oscillator and the perturbation is rank- $N$. In Section IV we investigate Combescure's conjecture, the answer provided by Bourget and the link to number theory and Vinogradov's conjecture. Finally, in Section V, we extend Milek and Seba's work to the rank$N$ case, correcting a number of subtle errors. We emphasise that their work has only recently been fully justified (by Bourget in [8]). We provide a complimentary justification, linked to the number-theoretic investigations and Vinogradov's conjecture just mentioned.

\section{Rank-N generalisation of Combescure's first the- orem}

We consider the measures

$$
m_{k, \lambda_{k}}=\left\langle\psi_{k}\left|E_{\lambda_{k}}(S)\right| \psi_{k}\right\rangle
$$

\footnotetext{
${ }^{2}$ The reference is to the 1954 English translation of Vinogradov's original work, published in 1947. The work in Vinogradov's 1947 monograph incorporates results from a series of papers and a first monograph from 1937. It is unknown to us when the conjecture referred to was first presented, but it was at least fifty years ago.
} 
Each $\left|\psi_{k}\right\rangle$ admits a cyclic subspace of $\mathcal{H}, \mathcal{H}_{k}$. As argued in the later part of the proof of Theorem 4.3 in [19], on the space $\mathcal{H} \ominus\left(\bigoplus_{k=1}^{N} \mathcal{H}_{k}\right)$, the perturbation

$$
\sum_{k=1}^{N} \lambda_{k}\left|\psi_{k}\right\rangle\left\langle\psi_{k}\right|
$$

is null and thus $V=U$ is trivially pure point. Henceforth, we may safely restrict our proof to the subspace $\bigoplus_{k=1}^{N} \mathcal{H}_{k}$ for which the vectors $\left|\psi_{k}\right\rangle$ form a cyclic set.

Directly following Combescure, the measure for a point $x \in[0,2 \pi)$ for the operator $V$ acting on the state $\left|\psi_{k}\right\rangle$ is given by

$$
m_{k, \lambda_{k}}(\{x\})=\frac{-4\left(1+\mu_{k}\right)}{\mu_{k}^{2}} B_{k}(x),
$$

where

$$
\mu_{k}=e^{i \lambda_{k} / \hbar}-1
$$

and

$$
B_{k}(x)=\left[\int_{0}^{2 \pi} d m_{k, \lambda_{k}=0}(\theta)\left(\sin ^{2}[(x-\theta) / 2]\right)^{-1}\right]^{-1} .
$$

This result is the essence of Lemma 1 in [15]. When $H_{0}$ is pure point, it is a trivial calculation to show that

$$
B_{k}^{-1}(x)=\sum_{n=0}^{\infty} \frac{\left|\left(a_{k}\right)_{n}\right|^{2}}{\sin ^{2}\left[\left(x-\theta_{n}\right) / 2\right]} .
$$

Corollary 2 in [15] is replaced with the following.

THEOREM II.1 Assume $H_{0}$ is pure point, with $\left\{\phi_{n}\right\}_{n \in \mathbb{N}}$ and $\left\{\alpha_{n}\right\}_{n \in \mathbb{N}}$ as eigenstates and eigenvalues. Let each

$$
\psi_{k}=\sum_{n=0}^{\infty}\left(a_{k}\right)_{n} \phi_{n}
$$

be cyclic for $H_{0}$ (hence, cyclic for $U$ and $V$ ) on $\mathcal{H}_{k}$ and $\left\langle\psi_{k} \mid \psi_{l}\right\rangle=\delta_{k l}$. Then $e^{i x}$ belongs to the point spectrum of $V_{\lambda_{1}, \ldots, \lambda_{N}}$ if and only if

$$
\prod_{k=1}^{N} B_{k}^{-1}(x)<\infty
$$

where

$$
\theta_{n}=2 \pi\left\{\alpha_{n} / 2 \pi \hbar\right\}
$$

$\{z\}$ being the fractional part of $z$. 
Proof. (II.1) The proof follows that in [15]. By the cyclicity of each $\left|\psi_{k}\right\rangle$ on $\mathcal{H}_{k}$ and the argument in Theorem 4.3 of [19], $e^{i x}$ is an eigenvalue of $V_{\lambda_{1}, \ldots, \lambda_{N}}$ if and only if every $m_{k, \lambda_{k}}(\{\theta\}) \neq 0$ at $\theta=x$. As already mentioned, using

$$
d m_{k, \lambda_{k}=0}=\sum_{n=0}^{\infty}\left|\left(a_{k}\right)_{n}\right|^{2} \delta\left(\theta-\theta_{n}\right) d \theta
$$

we obtain, for each $k$,

$$
B_{k}^{-1}(x)=\sum_{n=0}^{\infty} \frac{\left|\left(a_{k}\right)_{n}\right|^{2}}{\sin ^{2}\left[\left(x-\theta_{n}\right) / 2\right]} .
$$

We now consider the eigenvalue $e^{i x}$. If it were to be that for some $k, m_{k, \lambda_{k}}(\{x\})=$ 0 , then we would have found a vector, namely $\left|\psi_{k}\right\rangle$, such that $V\left|\psi_{k}\right\rangle$ was continuous. We have in fact found that the whole subspace $\mathcal{H}_{k}$ is continuous. Thus, for $V$ to be pure point, we require every $m_{k, \lambda_{k}}(\{\theta\}) \neq 0$. Thus, we are lead to consider the requirement

$$
\prod_{k=1}^{N} B_{k}^{-1}(x)<\infty
$$

As in [15], the relationship

$$
\sum_{n=0}^{\infty}\left|\left(a_{k}\right)_{n}\right|^{2} \operatorname{cotg}\left(\frac{x-\theta_{n}}{2}\right)=\operatorname{cotg} \frac{\lambda_{k}}{2 \hbar}
$$

also holds for each $k$. To show (4), we consider each $k$ separately. The proof is the same as for the rank-1 case. See [15]. Points to consider are that each projection operator in the rank- $N$ projection is normalised and hence for every $k$ we have

$$
\sum_{n=0}^{N}\left|\left(a_{k}\right)_{n}\right|^{2}=1
$$

In order to complete the generalisation of Combescure's first theorem, we require, just as in [15], two additional Lemmas.

LEMMA II.2 If $\sum_{n=0}^{\infty}\left|\left(a_{k}\right)_{n}\right|<\infty$, then $B_{k}^{-1}(x)<\infty$ for almost every $x \in \mathbb{R}$.

For each $k \in 1, \ldots, N$, the proof is identical to that in [15]. 
LEMMA II.3 The following two statements are equivalent.

(a) For almost every $\left(\lambda_{1}, \ldots, \lambda_{N}\right), V_{\lambda_{1}, \ldots, \lambda_{N}}$ has only a point spectrum.

(b) For every $k \in\{1, \ldots, N\}$ and for almost every $x, B_{k}(x) \neq 0$.

The proof is again virtually identical to Combescure's proof. For each $k$, the continuous part of the spectrum is supported outside the set $E_{k}=\{x \in[0,2 \pi)$ : $\left.B_{k}(x) \neq 0\right\}$ and, for $\lambda_{k} \neq 0$, the point part of $d m_{k, \lambda_{k}}$ is supported by the set $E_{k}$. Thus, for $V_{\lambda_{1}, \ldots, \lambda_{N}}$ to be pure point for almost every $\lambda_{1}, \ldots, \lambda_{N}$ and for every $k$, we require

$$
m_{k, \lambda_{k}}\left([0,2 \pi) \backslash E_{k}\right)=0 .
$$

This in turn implies that for every $k$

$$
\int_{0}^{2 \pi} d \lambda_{k}^{\prime} h\left(\lambda_{k}^{\prime}\right) m_{k, \lambda_{k}}\left([0,2 \pi) \backslash E_{k}\right)=0
$$

where $\lambda_{k}^{\prime}=\lambda_{k} / \hbar$ and

$$
h(\lambda)=2 \Re \frac{1}{1-c e^{i \lambda}}
$$

for some $|c|<1$.

Lemma 5 in [15] trivially applies for each $k$. Thus, we have generalised Combescure's work to obtain the result that the Floquet operator for the rank- $N$ perturbed Hamiltonian has a pure point spectrum. The result matches that in [19].

\section{Rank-N generalisation of Combescure's second theorem}

Having shown that the Floquet operator remains pure point for perturbations constructed from the vectors $\left|\psi_{k}\right\rangle \in l_{1}\left(H_{0}\right)$, Combescure relaxes this condition to allow for the emergence of a continuous spectral component of the Floquet operator. This result is easily generalised to the rank- $N$ case. The key point is that the technique in [15] applies independently for each $k$. We do not discuss the details of the rank-1 proof here at all, delaying an analysis to Section IV where we will have the opportunity to generalise the result still further. Here, we simply provide the argument for why each $k$ may be treated independently. Before proceeding, some subtleties of what Combescure actually shows are highlighted. They are seemingly overlooked by some in the literature (e.g., [21]).

The cyclicity requirement was essential in the proof that the Floquet operator spectrum was pure point. Here, we can happily ignore the cyclicity conditions, as our only goal is to establish the existence of a state in the continuous subspace 
$\mathcal{H}_{\text {cont }}$. We need not try and ensure the result obtained by considering $\langle\psi|E(S)| \psi\rangle$ is applicable to all other vectors in $\mathcal{H}$-the very idea is ill-formed as the perturbation is null on a subset of $\mathcal{H}$ and thus there is always part of $\mathcal{H}$ where $V$ has a discrete spectrum. Milek and Seba seem to have missed this point, restating Theorem 1 in [15] in a way that implies that all $\psi$ are in $\mathcal{H}_{\text {cont }}$.

If

$$
\left\langle\psi_{k}|E(\{x\})| \psi_{k}\right\rangle=0
$$

then $\mathcal{H}_{a c}$ contains at least the state $\left|\psi_{k}\right\rangle$. The point to be mindful of is that this does not allow one to conclude that the Hilbert space for the operator $V$ has $\mathcal{H}_{p p}=\emptyset$, as implied by Milek and Seba [21]. To draw that conclusion would require an argument to show that a cyclic vector does in fact exist for $V$. This does not seem possible in the general context we have here.

Combescure's proof (Lemma 6 in [15]) that $\sigma_{\text {cont }}(V) \neq \emptyset$ is based on showing that $B^{-1}(x) \rightarrow \infty$ (equation (3)). As the spectral measure of a single point $x$ is proportional to $B(x)$ (equation (2)), if $B^{-1}(x) \rightarrow \infty$, then the contribution of the single point is zero. That is, $e^{i x}$ is in the continuous spectrum of the Floquet operator. Combescure argues (see Section IV for details) that

$$
B^{-1}(x) \geq \# S(x)
$$

where $\# S(x)$ is the number of element of a particular set $S$. She then shows (the bulk of the proof) that $\# S(x) \rightarrow \infty$ and thus $B^{-1}(x) \rightarrow \infty$. We generalise the result in a straightforward manner.

THEOREM III.1 Assume $\alpha_{n}=n \hbar \omega$ with $\omega$ irrational. If $\left|\psi_{k}\right\rangle \notin l_{1}\left(H_{0}\right)$ for at least one $k \in 1, \ldots, N$, then $\sigma_{\text {cont }}(V) \neq \emptyset$.

Proof. (III.1) Following the same argument as for the rank-1 case, we take

$$
\left|\left(a_{k}\right)_{n}\right|=n^{-\gamma} 2 \pi
$$

for the state $\left|\psi_{k}\right\rangle$, in such a way that the condition $\left\langle\psi_{k} \mid \psi_{l}\right\rangle=\delta_{k l}$ is preserved.

With this construction, the proof that the number of elements in $S(x)$ is infinite [15] applies to each subsequence $S_{k}(x)$. The number of elements, $\# S_{k}(x)$, in each sub-sequence for which $\left|p s i_{k}\right\rangle \notin l_{1}\left(H_{0}\right)$, is infinite. The Floquet operator for the rank- $N$ perturbed harmonic oscillator obtains a continuous spectral component.

\section{III.1 Discussion}

It must be noted that the proof of Lemma 6 in [15] is only valid for the eigenvalue spectrum,

$$
\alpha_{n}=n \hbar \omega
$$


of the harmonic oscillator. Combescure does however conjecture that the argument will be valid for more general eigenvalue spectra, including the rotor

$$
\alpha_{n} \propto n^{2}
$$

For Milek and Seba's numerical work (using the rotor) to be based on valid mathematical arguments, a proof of this conjecture is required. It was only very recently that a proof was developed [8], some fifteen years after the numerical results of Milek and Seba were published. As already mentioned, the work presented here, aiming to justify Milek and Seba's numerical work, was developed independently and is complimentary to Bourget's approach.

In Section IV we show that if a conjecture from number theory on the estimation of exponential sums is true, then Milek and Seba's work can be justified. The rank- $N$ generalisation is straightforward. Considering the number theory conjecture has stood for some fifty years, it seems we may have to wait quite some time for a proof. ${ }^{3}$

For more general eigenvalue spectra (loosely $\alpha_{n} \propto n^{j}$ ) the situation is similar. For $j \geq 3$ Bourget [7] made significant progress. He has now covered the $j=2$ case [8]. A continuous component of the Floquet operator exists for certain constructions of $|\psi\rangle$. The conditions, for all $j \geq 2$, are complicated and more restrictive than the $|\psi\rangle \notin l_{1}\left(H_{0}\right)$ condition for the harmonic oscillator. The result is easily extended to the rank- $N$ case due to the independence of each $k$ as already discussed. Here, by utilising a number-theoretic conjecture, we will provide improvements to the work of Bourget (both the $j \geq 3$ and $j=2$ cases). See Section IV.

Returning to the harmonic oscillator case, by applying Theorem III.1 we may conclude that for each $\left|\psi_{k}\right\rangle \notin l_{1}\left(H_{0}\right), \mathcal{H}_{k}$ is purely continuous. Thus, by dropping the $l_{1}$ condition for all $\left|\psi_{k}\right\rangle$, we have shown that $V$ is purely continuous on the subspace of $\mathcal{H}$ where the perturbation is non-zero. On the subspace of $\mathcal{H}$ where the perturbation is zero, $V=U$ trivially and thus that portion of the Hilbert space remains pure point.

\section{Discussion of Combescure's conjecture}

Combescure [15] makes a remark (Remark c.) that she believes Theorem III.1 (Lemma 6, [15]) is generalisable to include systems other than the harmonic oscillator. Explicitly, she conjectures that Hamiltonians, $H_{0}$, with eigenvalues, $\alpha_{n}$,

\footnotetext{
${ }^{3}$ Of course, now that Bourget has provided a direct proof for the rotor, the numerical simulations are on safe ground.
} 
of the form

$$
\alpha_{n}=\hbar \sum_{j=0}^{p} \beta_{j} n^{j}
$$

with $\beta_{j} T / 2 \pi$ Diophantine for some $j: 1 \leq j \leq p$ will have the vector $\psi$ in the continuous spectral subspace of $V_{\lambda}$.

At an intuitive level, one would expect this to be true. The precise nature of the eigenvalue spectra (proportional to $n$ or a polynomial in $n$ ) should not make a significant difference. Milek [20] argues that Combescure's work can be used in the $n^{2}$ case based on evidence from some numerical work that shows that the sequences obtained are "almost random"- - however, the argument is not entirely convincing to us. The cited numerical work of Casati et. al. [10] discusses the existence of correlations in the energy levels, rather than the lack of correlations. While the deviations from a Poisson distribution look small to the naked eye, Casati et. al. [10] find deviations from the expected Poisson distribution of up to 17 standard deviations. The energy levels are correlated-it is arguable that they are not characterisable as "almost random" as Milek asserts.

In late 2002, Bourget [7] produced a proof of a slightly modified conjecture for all but the $p=2$ case in (5). The techniques used by Bourget are similar to those followed in this work. We will analyse Bourget's work, and highlight the key breakthrough made. We also provide a modified argument to obtain the proof which is, we believe, significantly easier to follow. Importantly, it also covers the $p=2$ case, unresolved by Bourget (until very recently) due to technical difficulties. However, it comes at the expense of relying upon a conjecture. Our result plays a complementary role in understanding, or perhaps appreciating, Bourget's proof. The reliance on the conjecture removes the need for much of the technical wizardry in Bourget's proof, and also strengthens the work. Our analysis also indicates, or highlights, that Combescure's conjecture is solved by a numbertheoretic conjecture that has stood for over fifty years. What seems a perfectly reasonable conjecture on physical grounds is shown to be directly related to an abstract mathematical conjecture.

In what follows, we rely heavily upon the lemmas and theorems in Chapter 2 of [18]. We also use some results on Weyl sums from [22]. Of key importance is an understanding of the proof of Lemma 6 in [15] on the emergence of a continuous spectrum for the kicked harmonic oscillator. This will be discussed at the appropriate time in this section.

\section{IV.1 Number theory}

To discuss the conjecture, we require two concepts from number theory-the classification of irrational numbers and the discrepancy of a sequence. We first intro- 
duce the concepts and define the relevant ideas. We then proceed to analyse the conjecture and the proof provided by Bourget. As the discussion progresses, the new work that we have done will be presented.

For any number $\beta$, we define

- $[\beta]$, the integer part of $\beta$,

- $\{\beta\}$, the fractional part of $\beta$, and

- $\langle\beta\rangle=\min (\{\beta\}, 1-\{\beta\})$.

$\langle\beta\rangle$ is simply the "distance to the nearest integer". Definition IV.1 is taken directly from Kuipers and Niederreiter (Definition 3.4, p. 121, [18]).

DEFINITION IV.1 Let $\eta$ be a positive real number or infinity. The irrational $\beta$ is of type $\eta$ if $\eta$ is the supremum of all $\tau$ for which

$$
\underline{\lim _{n \rightarrow \infty}} q^{\tau}\langle q \beta\rangle=0,
$$

where $q$ runs through the positive integers.

The idea behind this definition can be seen by considering rational $\beta=p / q^{\prime}$ for some $p$ and $q^{\prime}$. Run through the positive integers $q$. At $q=q^{\prime},\langle q \beta\rangle=0$, so there is no supremum $\eta$ for $\tau$ in (6). In effect, $\eta \rightarrow \infty$. For irrational $\beta$, $\langle q \beta\rangle$ is never equal to zero but will approach zero. If the approach is very slow, then a small $\tau$ is enough to prevent (6) from approaching zero. $\langle q \beta\rangle$ approaching zero slowly is, in a sense, indicative of $\beta$ being badly approximated by rational numbers. Even for very large $q^{\prime}, p / q^{\prime}$ remains a poor approximation to $\beta$. Thus, the smaller $\eta$, the stronger the irrationality of $\beta$. This is reasonable in the sense that rational $\beta$ s act like numbers with $\eta \rightarrow \infty$. As stated in [18], all numbers $\beta$ have type $\eta \geq 1$.

We now define the discrepancy of a sequence-a measure of the non-uniformity of the sequence. We consider a sequence of numbers ${ }^{4} x_{n}$ in $[0,1)$

$$
\omega=\left(x_{n}\right)_{n \in \mathbb{N}} \text { with } x_{n} \in[0,1) .
$$

For $0 \leq a<b \leq 1$ and positive integer $N, A([a, b), N)$ counts the number of terms of the sequence (up to $x_{N}$ ) contained in the interval $[a, b)$,

$$
A([a, b), N)=\#\left\{n \leq N: x_{n} \in[a, b)\right\} .
$$

\footnotetext{
${ }^{4}$ Equivalently, consider any sequence $x_{n}$ and consider the discrepancy of the sequence modulo 1 .
} 
DEFINITION IV.2 The discrepancy $D_{N}$ of the sequence $\omega$ is

$$
D_{N}(\omega)=\sup _{0 \leq a<b \leq 1}\left|\frac{A([a, b), N)}{N}-(b-a)\right| .
$$

If the sequence $\omega$ is uniformly distributed in $[0,1)$ then $D_{N} \rightarrow 0$ as $N \rightarrow \infty$. In this case, every interval $[a, b]$ in $[0,1)$ gets its "fair share" of terms from the sequence $\omega$.

Estimating the discrepancy of a sequence will turn out to be vital in the analysis of Combescure's work. The sequence of interest is basically the eigenvalue sequence for $H_{0}$, but we will discuss this in greater detail later.

The starting point for the estimations that we require is (equation (2.42), Chapter 2, [18]). This is a famous result obtained by Erdös and Turán. It states that

$$
D_{N} \leq C\left(\frac{1}{m}+\sum_{h=1}^{m} \frac{1}{h}\left|\frac{1}{N} \sum_{n=1}^{N} e^{2 \pi i h x_{n}}\right|\right)
$$

for any real numbers $x_{1} \ldots x_{N}$ and any positive integer $m$. The sum

$$
S=\sum_{n=1}^{N} e^{2 \pi i h x_{n}}
$$

is an example of a class of exponential sums known as Weyl sums, reflecting the pioneering work of Weyl on providing estimations for them. Vinogradov [22] improved on some of the estimations of Weyl. Weyl and Vinogradov's results concern the modulus of the sum, $|S|$, and characterise it as

$$
|S| \leq \gamma N
$$

where $N$ is the number of terms in the sum and $\gamma$ tends to zero as $N \rightarrow \infty$. The subtle behaviour of $\gamma$ is linked to the rational/irrational nature of the terms in the sequence.

We will use some basic results from the introductory chapter of [22]. In general, we write

$$
S=\sum_{n=1}^{N} \exp (2 \pi i F(n))
$$

for some function $F(n)$. The application here is when

$$
F(n)=\beta n^{j} .
$$

For $\beta$ rational (not the case we will be interested in) L. K. Hau proved that $|S|$ was of order

$$
N^{1-(1 / j)+\epsilon}
$$


(page 3, [22]) and that this estimate could not be much improved. Here, we are interested in the case where $\beta$ is irrational. Estimations are much more difficult, and form the major aspect of the work by Vinogradov. The estimations depend upon making a rational approximation to $\beta$ and are complicated functions of $N$ and $j$. Very loosely, he obtains results like

$$
|S|=O\left(N^{1-\rho^{\prime}}\right)
$$

where

$$
\rho^{\prime}=\frac{1}{3(j-1)^{2} \log 12 j(j-1)} .
$$

Vinogradov states

It is a plausible conjecture that the estimate in (9) holds with $\rho^{\prime}$ replaced be $1 / j-\epsilon \ldots \dot{A}$ proof or disproof of this conjecture would be very desirable.

As the conjecture plays a central role in what follows, we state it formally.

CONJECTURE IV.3 Consider the sum

$$
S=\sum_{n=1}^{N} \exp 2 \pi i n^{j} \beta_{j} .
$$

For all $N$ greater than some critical value, we have

$$
|S| \leq c N^{1-(1 / j)+\epsilon}
$$

for all $\epsilon>0$ and some constant $c \in \mathbb{R}$.

We do not attempt to prove Conjecture IV.3. Given the lengths gone to by Vinogradov to obtain the results presented above, it seems rather unlikely that a proof or disproof will be found any time soon. ${ }^{5}$

\section{IV.2 Upper and lower bounds on discrepancy}

Armed with the estimations on Weyl sums, we now proceed to derive both upper and lower bounds on the discrepancy for sequences of the type

$$
\omega_{j}=\left(n^{j} \beta\right)
$$

\footnotetext{
${ }^{5}$ Incremental improvements on the estimations presented by Vinogradov in [22] have been made over time. While Bourget [7,8] makes use of these improved results, the conjecture itself remains unproven which is the only result of any consequence in this discussion.
} 
for $\beta$ of any type $\eta \geq 1$. It must be remembered that the upper bound obtained is contingent upon Conjecture IV.3. The lower bound obtained is not dependent upon any unproved conjectures. The result obtained highlights the "best possible" nature of the conjectured upper bound.

Firstly, (Lemma 3.2, p. 122, [18]) is generalised to arbitrary $j$.

LEMMA IV.4 The discrepancy $D_{N}\left(\omega_{j}\right)$ of $\omega_{j}=\left(n^{j} \beta\right)$ satisfies

$$
D_{N}\left(\omega_{j}\right) \leq C\left(\frac{1}{m}+N^{1-(1 / j)+\epsilon} c^{\prime} \sum_{h=1}^{m} \frac{1}{h\langle h \beta\rangle}\right)
$$

for any positive integer $m$ and $\epsilon>0$, where $C$ and $c^{\prime}$ are absolute constants.

Proof. (IV.4) Consider (8). It is applicable to the first $N$ terms of the sequence $\omega_{j}$. We have

$$
D_{N}\left(\omega_{j}\right) \leq C\left(\frac{1}{m}+\frac{1}{N} \sum_{h=1}^{m} \frac{1}{h}\left|\sum_{n=1}^{N} e^{2 \pi i h n^{j} \beta}\right|\right)
$$

for any positive integer $m$. Consider the sum over $n$,

$$
\left|\sum_{n=1}^{N} e^{2 \pi i h n^{j} \beta}\right|
$$

Conjecture IV.3 allows this sum to be bounded by

$$
c N^{1-(1 / j)+\epsilon} .
$$

We are free to write

$$
c=\frac{c^{\prime}}{|\sin \pi h \beta|}
$$

as $\sin \pi h \beta$ is just some positive real number. Substituting this result into (10), we obtain

$$
D_{N}\left(\omega_{j}\right) \leq C\left(\frac{1}{m}+N^{-(1 / j)+\epsilon} c^{\prime} \sum_{h=1}^{m} \frac{1}{h} \frac{1}{|\sin \pi h \beta|}\right) .
$$

Now following the argument at the end of (Lemma 3.2, [18]) the desired result is obtained.

We now give the generalisation of (Theorem 3.2, [18]). It provides the "best" upper bound one could hope for when estimating the discrepancy of the sequence $\omega_{j}=\left(n^{j} \beta\right)$. Again, remember that the proof relies on Conjecture IV.3. 
TheOrem IV.5 Assume Conjecture IV.3 is true. Let $\beta$ be of finite type $\eta$. Let $j$ be a positive integer $j \geq 1$. Then, for every $\epsilon>0$, the discrepancy $D_{N}\left(\omega_{j}\right)$ of $\omega_{j}=\left(n^{j} \beta\right)$ satisfies

$$
D_{N}\left(\omega_{j}\right)=O\left(N^{-1 /(\eta j)+\epsilon}\right) .
$$

Proof. (IV.5) Let $\epsilon>0$ be fixed. By (Lemma 3.1 and Lemma 3.3, p. 121-3, [18]),

$$
\sum_{h=1}^{m} \frac{1}{h\langle h \beta\rangle}=O\left(m^{\eta-1+\epsilon^{\prime}}\right)
$$

for a fixed $\epsilon^{\prime}>0$. Combining this with Lemma IV.4, gives

$$
D_{N}\left(\omega_{j}\right) \leq C\left(\frac{1}{m}+N^{-(1 / j)+\epsilon^{\prime \prime}} m^{\eta-1+\epsilon^{\prime}}\right)
$$

for all $m \geq 1$. Now choose $m=\left[N^{1 /(\eta j)}\right]$. We obtain

$$
\begin{aligned}
D_{N}\left(\omega_{j}\right) & \leq C\left(N^{-1 /(\eta j)}+N^{-(1 / j)+\epsilon^{\prime \prime}+(1 / j)-1 /(\eta j)+\epsilon^{\prime} /(\eta j)}\right) \\
& =O\left(N^{-1 /(\eta j)+\epsilon}\right)
\end{aligned}
$$

where $\epsilon=\epsilon^{\prime \prime}+\epsilon^{\prime} /(\eta j)$.

Theorem IV.5 is, in a sense, optimal. For functions $f, g$, define $f=\Omega(g)$ if $f / g \nrightarrow 0$.

THEOREM IV.6 Let $\beta$ be of finite type $\eta$. Let $j$ be a positive integer $j \geq 1$. Then, for every $\epsilon>0$, the discrepancy $D_{N}\left(\omega_{j}\right)$ of $\omega_{j}=\left(n^{j} \beta\right)$ satisfies

$$
D_{N}\left(\omega_{j}\right)=\Omega\left(N^{-1 /(\eta j)-\epsilon}\right) .
$$

Proof. (IV.6) Let $\epsilon>0$ be fixed. For any given $\epsilon^{\prime}>0$, there exists $0<$ $\delta<\eta$ with $1 /(\eta-\delta)=(1 / \eta)+\epsilon^{\prime}$. By (Definition 3.4, p. 121, [18]), we have $\underline{\lim }_{q \rightarrow \infty} q^{\eta-(\delta / 2)}\langle q \beta-j\rangle=0$ and thus

$$
\left\langle q \beta_{j}\right\rangle<q^{-\eta+(\delta / 2)}
$$

for an infinite number of positive integers $q$. There are infinitely many positive integers $q$ and $p$ such that

$$
|\beta-p / q|<q^{-1-\eta+(\delta / 2)} .
$$

That is, by choosing $q$ large enough, we can always find a $p$ such that $|q \beta-p|=$ $\langle q \beta\rangle$. As $q$ increases $p / q$ is a better approximation to the irrational $\beta$. For $\theta$ some irrational with $|\theta|<1$, we have

$$
\beta=p / q+\theta q^{-1-\eta+(\delta / 2)} .
$$


Pick a $q$ such that the above relations are valid. Set

$$
N=\left[q^{j(\eta-\delta)}\right]
$$

Then for $1 \leq n^{j} \leq N^{1 / j}$,

$$
n^{j} \beta=n^{j}(p / q)+\theta_{n},
$$

with

$$
\begin{aligned}
\left|\theta_{n}\right| & =\left|n^{j} \theta q^{-1-\eta+(\delta / 2)}\right| \\
& <n^{j} q^{-1-\eta+(\delta / 2)} \\
& \leq q^{[j(\eta-\delta)]^{1 / j}-1-\eta+(\delta / 2)} \\
& =q^{-1-(\delta / 2)} .
\end{aligned}
$$

Thus, none of the fractional parts $\{\beta\},\left\{2^{j} \beta\right\}, \ldots,\left\{\left[N^{1 / j}\right] \beta\right\}$ lie in the interval $J=\left[q^{-1-(\delta / 2)}, q^{-1}-q^{-1-(\delta / 2)}\right)$, so

$$
D_{N}\left(\omega_{j}\right) \geq\left|\frac{A(J, N)}{N}-\lambda(J)\right|=\lambda(J)
$$

where $\lambda(J)$ is simply the "size" of the set $J$. For large enough $q$ we have $\lambda(J) \geq$ $1 / 2 q$. But from the definition of $N$ it is clear that

$$
N \leq q^{j(\eta-\delta)} \leq N+1 \leq 2 N,
$$

so

$$
q^{-1} \geq c N^{-[j(\eta-\delta)]^{-1}}
$$

Combining these inequalities, we obtain

$$
\begin{aligned}
D_{N}\left(\omega_{j}\right) & \geq c^{\prime} N^{-[j(\eta-\delta)]^{-1}} \\
& =c^{\prime} N^{-(1 / j)(1 /(\eta-\delta))} \\
& =c^{\prime} N^{-(1 / j)\left((1 / \eta)+\epsilon^{\prime}\right)} \\
& =c^{\prime} N^{-1 /(\eta j)-\epsilon}
\end{aligned}
$$

where $\epsilon=\epsilon^{\prime} / j$. That is, we have shown, for all $\epsilon>0$, that

$$
D_{N}\left(\omega_{j}\right)=\Omega\left(N^{-1 /(\eta j)-\epsilon}\right) .
$$




\section{IV.3 Combescure's conjecture, Bourget's work and new results}

Before discussing the conjecture, we must clearly understand Combescure's proof for the harmonic oscillator case. As stated in Section III, the aim is to show that

$$
B^{-1}(x)=\sum_{n=0}^{\infty}\left|a_{n}\right|^{2}\left(\frac{2}{\sin \left(x-\theta_{n}\right)}\right)^{2} \rightarrow \infty .
$$

We define the set $S(x)$

$$
S(x)=\left\{n:\left|x-\theta_{n}\right| \leq\left|a_{n}\right|=n^{-\gamma} 2 \pi\right\} .
$$

Each $n$ is an element of $S(x)$ if $x$ is "close to $\theta_{n}$ ". Note that $\theta_{n}=2 \pi\left\{\alpha_{n} / 2 \pi \hbar\right\}$, where $\{$.$\} is the fractional part, not "set" and \alpha_{n}$ are the eigenvalues of the base Hamiltonian $H_{0}$.

Given that $\sin x \leq x$ for all $x \geq 0$, a lower bound for $B^{-1}(x)$ is obtained,

$$
\begin{aligned}
B^{-1}(x) & \geq \sum_{n=0}^{\infty}\left|a_{n}\right|^{2}\left(\frac{2}{x-\theta_{n}}\right)^{2} \\
& \geq \sum_{n \in S(x)} \frac{4\left|a_{n}\right|^{2}}{\left(x-\theta_{n}\right)^{2}} \geq 4 \# S(x) .
\end{aligned}
$$

Each $n \in S(x)$ gives a contribution to the sum of greater than one as $\left|a_{n}\right| / \mid x-$ $\theta_{n} \mid \geq 1$. By only considering \#S(x), we simply count a " 1 " each time.

The results on discrepancy of sequences are now used, with the sequence $\omega_{\mathrm{HO}}=\left(\theta_{n} / 2 \pi\right)$. Note that each element of the sequence $\omega_{\mathrm{HO}}$ is in $[0,1)$.

Consider the interval, defined for every $x \in(0,2 \pi)$ and centred around $x / 2 \pi$,

$$
J_{N}(x)=\left[\frac{x}{2 \pi}-N^{-\gamma}, \frac{x}{2 \pi}+N^{-\gamma}\right] .
$$

For large enough $N, J_{N}(x) \subset[0,1)$. The size of the interval is $2 N^{-\gamma}$. Using this particular subset and noting that the definition of discrepancy (7) involves taking the supremum over all subsets of $[0,1)$, Combescure obtains

$$
\left|N^{-1} A\left(J_{N}(x), N\right)-2 N^{-\gamma}\right| \leq D_{N}\left(\omega_{\mathrm{HO}}\right) .
$$

Multiplying through by $N$ gives

$$
\left|A\left(J_{N}(x), N\right)-2 N^{1-\gamma}\right| \leq N D_{N}\left(\omega_{\mathrm{HO}}\right) .
$$

As $|\psi\rangle \notin l_{1}\left(H_{0}\right)$

$$
\sum\left|a_{n}\right| \rightarrow \infty
$$


and thus

$$
1 / 2<\gamma \leq 1
$$

from simple convergence arguments. Therefore, $N^{1-\gamma}$ grows at a rate ${ }^{6}$ less than $N^{1 / 2}$. At this stage, Combescure utilises the theorems discussed above on the discrepancy of sequences. For the eigenvalue sequence, $\alpha_{n}=n \hbar \omega$, of the harmonic oscillator ${ }^{7}$ the $j=1$ case of Theorem IV.5 applies which is exactly (Theorem 3.2, [18]). Combescure obtains the result ${ }^{8}$

$$
D_{N}\left(\omega_{\mathrm{HO}}\right)=O\left(N^{-1(/ \eta)+\epsilon}\right) .
$$

For the sequence $\omega_{\mathrm{HO}}, \beta=\omega / 2 \pi$. If $\beta$ is an irrational of constant type $(\eta=1)$, the strongest type of irrational, then

$$
N D_{N}\left(\omega_{\mathrm{HO}}\right)=O\left(N^{\epsilon}\right)
$$

As the right-hand side of (14) can be made to grow arbitrarily slowly, we conclude that the left-hand side must grow slowly too. Thus, to cancel out the growth of $2 N^{1-\gamma}, A\left(J_{N}(x), N\right)$ must grow at a rate arbitrarily close to that of $2 N^{1-\gamma}$. We see that

$$
A\left(J_{N}(x), N\right) \rightarrow \infty
$$

as $N \rightarrow \infty$. It is now a simple observation [15] that this implies that $\# S(x) \rightarrow \infty$ and thus $B^{-1}(x) \rightarrow \infty$. Thus, $e^{i x}$ is in the continuous spectral subspace of the Floquet operator $V$.

The importance of the eigenvalue sequence is seen in that if we cannot limit the right-hand side of (14), then we cannot place a lower limit on $A\left(J_{N}(x), N\right)$ and thus we cannot conclude that $B^{-1}(x) \rightarrow \infty$. Two barriers to limiting the right-hand side of this equation exist- $j$ and $\eta$. If, still in the harmonic oscillator case, we wished for $\beta=\omega / 2 \pi$ to only be of a weaker type, say $\eta=2$, we would no longer be able to conclude that $B^{-1} \rightarrow \infty$. The right-hand side would grow like $N^{(1 / 2)+\epsilon}$, which is always faster than $2 N^{1-\gamma}$ for $1 / 2<\gamma \leq 1$ which grows at a rate of $N^{(1 / 2)-\epsilon}$. Thus, no suitable lower limit for $A\left(J_{N}(x), N\right)$ can be found. Similarly, if the eigenvalue sequence is generalised (Combescure's conjecture)

\footnotetext{
${ }^{6}$ Interestingly, it can in fact not grow at all $(\gamma=1)$ which is a subtle point seemingly missed by Combescure and others. The rank- 1 projection operator from the vector $|\psi\rangle$ constructed with $\gamma=1$ is not shown to lead to the emergence of a continuous spectrum. Therefore, the statement that $|\psi\rangle \notin l_{1}\left(H_{0}\right)$ implies $|\psi\rangle \in \mathcal{H}_{\text {cont }}$ is not in fact proved to be true. There are vectors not in $l_{1}\left(H_{0}\right)$ that may not be in the continuous spectrum. In practice (numerical, experimental work) this should not cause any trouble. It is clearly easy to avoid $\gamma=1$.

${ }^{7}$ Do not confuse $\omega$, the harmonic oscillator frequency, with $\omega_{\mathrm{HO}}$, the label for the sequence in $[0,1)$, the discrepancy of which is being bounded.

${ }^{8}$ This is not based on a conjecture as for $j=1$ a direct proof is possible, bypassing Conjecture IV.3. See [18].
} 
then we run into trouble. For $j=2$, the lowest possible growth rate for the righthand side we can obtain, taking Conjecture IV.3 as true, applying Theorem IV.5 and noting Theorem IV.6 which says we cannot do any better, is , once again, $N^{(1 / 2)+\epsilon}$. For larger $j$, the situation only gets worse.

Given these seemingly significant problems, the natural question to ask is: "How does one get around this problem?". The answer is provided in the work of Bourget [7]. Bourget proves a weaker theorem than Combescure's conjecture. Where the same requirement on $|\psi\rangle$ is kept in [15], that it be in $l_{1}\left(H_{0}\right)$, Bourget has a $j$ dependent requirement. Essentially, for increasing $j$ the $a_{n}$ terms used to construct $|\psi\rangle$ must decrease more slowly with $n$. See Bourget's work for the exact requirement, which depends on the best estimates available for Weyl sums discussed earlier and thus is a non-trivial function of $j$.

The key insight in obtaining the proof is to modify the set $S(x)$ (equation (11)) and the corresponding interval $J_{N}(x)$ (equation (13)) that we consider. Importantly, they become $j$ dependent. Bourget reduces the shrinking rate of the set $J_{N}(x)$ as a function of $N$ just enough so as to allow the weaker limits on the discrepancy to be good enough to force the right-hand side of the equivalent to (14) to be less than the left-hand side, while keeping strong enough control on terms in the new set $S(x)$ to still argue that $B^{-1} \rightarrow \infty$.

Using the best available estimations on Weyl sums and plugging these into the upper bound formulas for discrepancy (as discussed above when introducing the work by Vinogradov), Bourget manages to provide a rigorous proof of the existence of a continuous spectral component of the Floquet operator (the essence of Combescure's conjecture) for $j \geq 3$ [7] and $j=2$ [8]. The proof is, unfortunately, unavoidably clouded by the "messy" estimates available for Weyl sums and thus, the essence of the proof is difficult to see. Here, we will revisit the proof, but (utilising Conjecture IV.3) apply Theorem IV.5 which says (using 2 $\epsilon$, rather than $\epsilon$ for technical reasons), for all $\epsilon>0$

$$
D_{N}(\omega)=O\left(N^{-1 /(\eta j)+2 \epsilon}\right) .
$$

With this very clean estimate, it is far easier to see how Bourget's work provides a proof that a continuous spectral component of the Floquet operator exists. It covers all $j \geq 2$. We highlight the fact that a solution to Vinogradov's conjecture provides an elegant solution to Combescure's physics conjecture. The $j$-dependence of the $a_{n}$ s used to construct $|\psi\rangle$ is straightforward.

THEOREM IV.7 Assume Conjecture IV.3 is true and thus Theorem IV.5 follows. Assume $\beta$ is irrational and of type $\eta$. Then for all positive integers, $j$, the Floquet operator, $V$, has $\sigma_{\text {cont }}(V) \neq \emptyset$ if $1 / 2<\gamma<1 / 2+1 /(2 \eta j)$.

Proof. (IV.7) The proof relies upon the techniques utilised by Bourget. In essence, we simply increase the size of the interval (equation (13)) from $2 N^{-\gamma}$ to 
$2 N^{2((1 / 2)-\gamma)}(\log N)^{-1 / 2}$. The important change is the first factor. The $\log N$ term is essential for technical reasons, but has a negligibly small effect on the shrinkage rate of the interval for large $N$. As $\log N / N^{4 \delta} \rightarrow 0$ as $N \rightarrow \infty$ for all $\delta>0$, for $N$ large enough we have

$$
2 N^{2((1 / 2)-\gamma)}(\log N)^{-1 / 2}>2 N^{2((1 / 2)-\gamma-\delta)} .
$$

Using this underestimate for the size of the interval, we easily obtain the equivalent of (14),

$$
\left|A\left(J_{N}(x), N\right)-2 N^{2(1-\gamma-\delta)}\right| \leq N D_{N}\left(\omega_{j}\right),
$$

for the sequence $\omega_{j}=\left(n^{j} \beta\right)$. Now, using Theorem IV.5, it is evident that to ensure $A\left(J_{N}(x), N\right) \rightarrow \infty$, we must have

$$
2(1-\gamma-\delta)>1-1 /(\eta j)+2 \epsilon
$$

or

$$
\gamma<1 / 2+1 /(2 \eta j)-\epsilon-\delta
$$

The condition

$$
1 / 2<\gamma<1 / 2+1 /(2 \eta j)
$$

where the " $<$ " sign has absorbed the arbitrarily small numbers $\epsilon$ and $\delta$, must be satisfied to force $A\left(J_{N}(x), N\right) \rightarrow \infty$.

Finally, we must show that $B^{-1}(x) \rightarrow \infty$ when this larger interval is used. Corresponding to the new interval $J_{N}(x)$, we introduce the new set $S(x)$,

$$
S(x)=\left\{n:\left|x-\theta_{n}\right| \leq 2 \pi N^{2((1 / 2)-\gamma)} \log N^{-1 / 2}\right\} .
$$

The estimate (12) is the same, except with the new set $S(x)$, which no longer has all terms greater than unity. Thus, it is not enough to simply count the number of terms in $S(x)$. A more subtle estimate is required. Replacing the numerator, $\left|a_{n}\right|$, with something smaller, $N^{-\gamma}$, and the denominator, $\left(x-\theta_{n}\right)$, with something larger, $2 \pi N^{2((1 / 2)-\gamma)} \log N^{-1 / 2}$, we obtain

$$
B^{-1}(x) \geq \frac{1}{\pi^{2}} \sum_{n \in S(x)} \frac{\log N}{N^{2(1-\gamma)}},
$$

which is essentially the estimate Bourget obtains. The estimate contained therein (Lemma 3.5 in [7]) then shows that $B^{-1}(x) \rightarrow \infty$ and the argument is complete.

Examining (15) we note that for $j=1$ (for $\eta=1$ ) we recover the simple result of Combescure. For all $j \geq 2$, we have a stronger ( $j$-dependent) condition 
on $|\psi\rangle$ than simply $|\psi\rangle \notin l_{1}\left(H_{0}\right)$. This complication is the main weakening of Combescure's conjecture that Bourget and we have been forced to make. Note that the restriction on $\gamma$ takes into account the end point subtleties referred to in the preceding discussions.

We have replaced the requirement that $|\psi\rangle \notin l_{1}\left(H_{0}\right)$ (i.e., $1 / 2<\gamma \leq 1$ ) with the $j$ dependent requirement $1 / 2<\gamma<1 / 2+1 /(2 j)$. In Bourget's work, the requirement is stronger - directly related to the replacement of the known limits on Weyl sums (in terms of $\rho$ in the earlier sections) with the "best possible" estimate from our Conjecture IV.3 of $(1 / j)-\epsilon$.

\section{IV.4 Summary}

Reliance on Conjecture IV.3 and the result of Theorem IV.5 derived from it has allowed us to discuss Bourget's proof without the complications of the messy estimations on Weyl sums. Bourget's proof is also $n$-dependent ( $m$ in his work) while ours is $n$-independent. This simplified discussion highlights the key aspects of Bourget's proof, both for $j \geq 3$ [7] and $j=2$ [8]. It has also shown that the emergence of a continuous spectral component of the Floquet operator is solved by Vinogradov's conjecture. A proof of Vinogradov's conjecture is no longer just of mathematical interest. It has a direct mathematical physics consequence.

Finally, note that the rank- $N$ equivalent of this work follows in the same way as presented for the harmonic oscillator case in Section III, providing a complete rank- $N$ generalisation of the work of Combescure [15].

\section{Generalising the results of Milek and Seba}

Having established that the continuous subspace of $\mathcal{H}, \mathcal{H}_{\text {cont }}$ is not empty, we wish to characterise it - by identifying the singular and absolutely continuous components. Here, we extend the result of Milek and Seba to rank- $N$ perturbations.

THEOREM V.1 Assume $H(t)$ is given by (1) and that (2) applies. Assume that $B_{k}^{-1}(x) \rightarrow \infty$ and thus $\mathcal{H}_{\text {cont }} \neq \emptyset$. Then $\mathcal{H}_{a c}=\emptyset$ and thus $\mathcal{H}_{s c}$ is not-empty. The Floquet operator, $V$, has a non-empty singular continuous spectrum.

Proof. (V.1) As shown in the proof of (Theorem II.4a, [19]) and easily calculated, the Floquet operator can be written in the form

$$
V=U+\sum_{k=1}^{N} R_{k}
$$


where

$$
R_{k}=\left(e^{i \lambda_{k} / \hbar}-1\right)\left|\psi_{k}\right\rangle\left\langle\psi_{k}\right| U .
$$

We can now use either (Theorem 5, [17]) or (Theorem 1, [6]). The theorem from the paper of Birman and Krein is more direct, so we use it here. It states that if we have two unitary operators, $U$ and $V$, that differ by a trace class operator, then the wave operators

$$
\Omega_{ \pm}=\mathrm{s}-\lim _{\nu \rightarrow \pm \infty} V^{\nu} U^{-\nu} P_{\mathrm{ac}}(U)
$$

exist and their range is the absolutely continuous subspace of $V$,

$$
R\left(\Omega_{ \pm}\right)=\mathcal{H}_{\mathrm{ac}}(V) .
$$

We must show that the difference $V-U$ is finite. With the notation in [19], where the perturbation $W$ is given by $A^{*} A$ and

$$
A=|\psi\rangle\langle\psi|,
$$

with

$$
|\psi\rangle=\sum_{n} a_{n} \phi_{n}
$$

we obtain

$$
\begin{aligned}
\operatorname{Tr} A^{*} A=\operatorname{Tr} A & =\sum_{l}\left\langle\phi_{l}|A| \phi_{l}\right\rangle \\
& =\sum_{l, m, n}\left\langle\phi_{l}\left|a_{n}\right| \phi_{n}\right\rangle\left\langle\phi_{m}\left|a_{m}^{*}\right| \phi_{l}\right\rangle \\
& =\sum_{l, m, n} a_{n} a_{m}^{*} \delta_{l n} \delta_{m l} \\
& =\sum_{l}\left|a_{l}\right|^{2}=1
\end{aligned}
$$

as $|\psi\rangle \in l_{2}\left(H_{0}\right)$ and is normalised. The perturbation to the Hamiltonian is trace class. The difference in unitary operators, $U$ and $V$, is also trace class. By the triangle inequality for norms,

$$
\left\|R_{k}\right\|_{\operatorname{Tr}} \leq\left\|\left(e^{i \lambda_{k} / \hbar}-1\right)\right\| \|\left|\psi_{k}\right\rangle\left\langle\psi_{k}\right|\left\|_{\operatorname{Tr}}\right\| U \|_{\operatorname{Tr}} .
$$

As $\|U\|_{T r}=1$,

$$
\begin{aligned}
\operatorname{Tr}\left(\sum_{k=1}^{N} R_{k}\right) & \leq \sum_{k} \|\left(e^{i \lambda_{k} / \hbar}-1 \| \sum_{l, m, n}\left\langle\phi_{l}\left|\left(a_{k}\right)_{n}\right| \phi_{n}\right\rangle\left\langle\phi_{m}\left|\left(a_{k}\right)_{m}^{*}\right| \phi_{l}\right\rangle\right. \\
& =\sum_{k}\left|e^{i \lambda_{k} \hbar}-1\right| \\
& =\sum_{k} \sqrt{2\left(1-\cos \lambda_{k} / \hbar\right)}
\end{aligned}
$$


Armed with a trace-class perturbation, we conclude that the wave operators exist. The existence of the operators $\Omega_{ \pm}$means that they are defined for all vectors in the Hilbert Space $\mathcal{H}$. Note (equation (17)) that the subspace $\mathcal{H}_{\mathrm{ac}}(V)$ is equal to the range of these operators. However, $P_{\mathrm{ac}}(U)$ gives zero when acting on any state in $\mathcal{H}$ because $U$ is pure point. Thus, $\mathcal{H}_{\mathrm{ac}}(V)$ is empty. As $\mathcal{H}_{\text {cont }}$ is not empty, $\mathcal{H}_{\mathrm{sc}}$ must be non-empty, and we have proved that a singular continuous subspace of the Floquet operator $V$ exists.

The key assumption in Theorem V.1 is that $B_{k}^{-1}(x) \rightarrow \infty$. This is certainly true for $j=1$ if $\left|\psi_{k}\right\rangle \neq l_{1}\left(H_{0}\right)$. For $j \geq 2$, Bourget [7,8] showed that one can construct vectors $\left|\psi_{k}\right\rangle$ for which $B_{k}^{-1}(x) \rightarrow \infty$. The results were discussed in detail in Section IV. We have shown, in Conjecture IV.7, that if Conjecture IV.3 is true then this result may be improved - the requirements on the states $\left|\psi_{k}\right\rangle$ are less restrictive. The result was also extended to rank- $N$ perturbations.

\section{V.1 Discussion}

Milek and Seba make a number of incorrect statements in obtaining this result for the rank-1 case. Firstly, they state that the operator ${ }^{9} R=[\exp (i \lambda / \hbar)-1]|\psi\rangle\langle\psi| U$ is rank-1 which it is not-the presence of the unitary operator $U$ stops $R$ from being rank-1. This is not, however, important. The applicability of the theorems in $[6,17]$ does not rely upon the rank of the operator $R$, but upon it being of traceclass. Secondly, they claim that the existence of the wave operators implies that

$$
\sigma_{\mathrm{ac}}(V) \subset \sigma_{\mathrm{ac}}(U) .
$$

This is, again, not true. Given that $\sigma_{\mathrm{ac}}(U)$ is empty, it is indeed possible to conclude that $\sigma_{\text {ac }}(V)$ is empty, as discussed above, but the relation (18) does not follow. Consider the situation where $\sigma_{\text {cont }}(U)$ is not empty. Then there is a set of vectors in $\mathcal{H}$ which are continuous for $U$. These vectors form the domain for the operator $V^{\nu}$ in the wave operators. The action with $V^{\nu}$ does not however keep us in the subspace $\mathcal{H}_{\text {cont }}(U)$ as the space we get to (the range for $V^{\nu}$ ) is only invariant for $\mathcal{H}_{\text {cont }}(V)$, not $\mathcal{H}_{\text {cont }}(U)$. Thus, we may obtain a vector, necessarily in $\mathcal{H}_{\text {cont }}(V)$ due to invariance, but possibly in $\mathcal{H}_{\mathrm{s}}(U)$, and thus, we cannot conclude that $\sigma_{\text {ac }}(V) \subset \sigma_{\text {ac }}(U)$. These two points discussed do not make the final results of Milek and Seba wrong, but "only" the proofs.

Of greatest concern is the use of Lemma 6 in [15] without justification. Milek and Seba have assumed that Combescure's conjecture is true. It has taken fifteen years, and a significant amount of work by Bourget, for that to be shown to be the case. We have shown that the conjecture is directly linked to a long standing

\footnotetext{
${ }^{9}$ As we are dealing with the rank-1 case, the subscript $k$ may be dropped from (16).
} 
number-theoretic conjecture. The work has also been extended to cover rank- $N$ perturbations.

\section{Summary}

We have generalised the work of both Combescure [15] and Milek and Seba [21] from rank-1 to rank- $N$. We have also discussed in detail Combescure's conjecture, our work on estimations of discrepancy and the demonstration by Bourget [7] that a continuous spectral component of the Floquet operator does exist for certain constructions of $|\psi\rangle$. This covers the essential aim of Combescure's conjecture on the existence of a continuous spectral component. A clear view of the essence of Bourget's proof has been provided by taking a reasonable number-theoretic conjecture to be true. With this clear view, the work of Bourget becomes more accessible. A resolution to Vinogradov's conjecture would have direct implications in mathematical physics.

An in depth critical analysis of the work of Milek and Seba was also undertaken; we highlighted a number of misconceptions in the work. A proof of Vinogradov's conjecture, allowing our work to provide an elegant solution to Combescure's conjecture, remains desirable.

\section{Acknowledgements}

This work was supported by the Australian Research Council. We thank Olivier Bourget for some useful discussions. One of the authors, J. McCaw, also wishes to acknowledge the financial support provided by the David Hay Memorial Fund.

\section{References}

[1] S. Albeverio and Ph. Blanchard, editors. Trends and Developments in the Eighties : Bielefeld Encounters in Mathematics and Physics IV and V, Singapore, 1985. World Scientific.

[2] S. Albeverio, G. Casati, and D. Merlini, editors. Stochastic Processes in Classical and Quantum Systems: proceedings of the 1st Ascona-Como International Conference, held in Ascona, Ticino (Switzerland), June 24-29, 1985, volume 262 of Lecture Notes in Physics, Berlin, 1986. SpringerVerlag. 
[3] W. F. Ames, E. M. Harrell II, and J. V. Herod, editors. Differential Equations with Applications to Mathematical Physics, volume 192 of Mathematics in Science and Engineering, Boston, 1993. Academic Press.

[4] I. Antoniou and Z. Suchanecki. Quantum systems with fractal spectra. Chaos Solitons Fractals, 14:799, 2002.

[5] I. Antoniou and Z. Suchanecki. On Computability of Decaying and Nondecaying States in Quantum Systems with Cantor Spectra. Int. J. Theor. Phys., 42(10):2255, 2003.

[6] M. Sh. Birman and M. G. Krein. On they theory of wave and scattering operators. Dokl. Akad. Nauk. SSSR, 144(3):475, 1962.

[7] Olivier Bourget. Singular continuous Floquet operator for systems with increasing Gaps. J. Math. Anal. App., 276:28, 2002.

[8] Olivier Bourget. Singular continuous Floquet operator for periodic quantum systems. J. Math. Anal. App., 301:65, 2005.

[9] Wesley E. Brittin and Lita G. Dunham, editors. Lectures in theoretical physics: lectures delivered at the Summer Institute for Theoretical Physics, University of Colorado, Boulder, 1958, volume 1 of Lectures in theoretical physics, New York, 1959. Interscience Publishers.

[10] G. Casati, B. V. Chirikov, and I. Guarneri. Energy-Level Statistics of Integrable Quantum Systems. Phys. Rev. Lett., 54(13):1350, April 1985.

[11] G. Casati and J. Ford, editors. Stochastic Behavior in Classical and Quantum Hamiltonian Systems. Volta Memorial Conference, Como, 1977, Berlin, 1979. Springer-Verlag.

[12] G. Casati, I. Guarneri, and U. Smilansky, editors. Caos Quantico, Proceedings of the International School of Physics "Enrico Fermi" ; course 119, Amsterdam, 1993. North-Holland.

[13] Joel E. Cohen, Harry Kesten, and Charles M. Newman, editors. Random matrices and their applications: proceedings of the AMS-IMS-SIAM Joint Summer Research Conference held June 17-23, 1984, with support from the National Science Foundation, volume 50 of Contemporary mathematics, 0271-4232, Providence, Rhode Island, 1986. Proc. Am. Math. Soc.

[14] J. M. Combes. Connections Between Quantum Dynamics and Spectral Properties of Time-Evolution Operators. In Ames et al. [3], page 59. 
[15] M. Combescure. Spectral Properties of a Periodically Kicked Quantum Hamiltonian. J. Statist. Phys., 59:679, 1990.

[16] Piotr Garbaczewski, Marek Wolf, and Aleksander Weron, editors. ChaosThe Interplay Between Stochastic and Deterministic Behaviour. Proceedings of the XXXIst Winter School of Theoretical Physics held in Karpacz, Poland, 13-24 February 1995, volume 457 of Lecture notes in physics, Berlin, 1995. Springer-Verlag.

[17] James S. Howland. Scattering Theory for Hamiltonians Periodic in Time. Indiana Univ. Math. J., 28(3):471, 1979.

[18] L. Kuipers and H. Niederreiter. Uniform Distribution of Sequences. John Wiley \& Sons, Inc, New York, 1974.

[19] James McCaw and B. H. J. McKellar. Pure point spectrum for the time evolution of a periodically rank-N kicked Hamiltonian. J. Math. Phys., 46:032108, 2005 .

[20] B. Milek. A kicked quantum system including the continuum. Phys. Lett. A, 135(1):1, February 1989.

[21] B. Milek and P. Seba. Singular continuous quasienergy spectrum in the kicked rotator with separable perturbation: Possibility of the onset of quantum chaos. Phys. Rev. A, 42(6):3213, September 1990.

[22] I. M. Vinogradov. The Method of Trigonometrical Sums in the Theory of Numbers. Interscience Publishers, London, 1954. 